\title{
Mapping Service Components to EJB Business Objects
}

\author{
Giacomo Piccinelli \\ HP Labs Bristol \\ Filton Rd, Stoke Gifford \\ Bristol BS34 8QZ, UK \\ giacomo_piccinelli@hp.com
}

\author{
Wolfgang Emmerich and Anthony Finkelstein \\ Dept. of Computer Science \\ University College London \\ Gower Street, London WC1E 6BT, UK \\ $\{$ w.emmerich|a.finkelstein\}@cs.ucl.ac.uk
}

\begin{abstract}
The emerging trends for e-business engineering revolve around specialisation and cooperation. Successful companies focus on their core competencies and rely on a network of business partners for the support services required to compose a comprehensive offer for their customers. Modularity is crucial for a flexible e-business infrastructure, but related requirements seldom reflect on the design and operational models of business information systems. Software components are widely used for the implementation of e-business applications, with proven benefits in terms of system development and maintenance. We propose a service-oriented componentisation of e-business systems as a way to close the gap with the business models they support. Blurring the distinction between external services and internal capabilities, we propose a homogeneous model for the definition of e-business applications components and present a process-based technique for component modelling. We finally present an Enterprise Java Beans extension that implements the model.
\end{abstract}

\section{Introduction}

E-business has attracted attention from software vendors, system integrators, solution providers, and ultimately from businesses. The traditional idea of e-business revolves around offering to customers, suppliers, and business partners the capability to automate their interaction with the sales or procurement department of a company. The Internet acts as an additional channel improving speed and automation for interaction processes. The e-service model [8] builds on the power of existing e-business capabilities and extends it with the aim of making the Internet a pervasive reality for businesses.

In the e-service model, any type of asset can be engineered and presented as a service to potential users inside and outside a company. The encapsulation of specific sets of business capabilities into well-defined service modules improves internal management and execution. Modularity helps localise points of weakness, over-sizing, under-sizing, and solve integration problems with other parts of the business infrastructure [9]. Modularity enables the outsourcing of specific business activities, as well as the external offer of excess capacity. The combination of the e-services model and enabling business infrastructures like electronic marketplaces gives a dynamic angle to internalisation and externalisation of service components. The focus shifts from the connection to a specific business partner, to the definition of a specific business need. The link with the business partner offering the best conditions for a service, at every point in time can be built by exploiting the aggregation power of open electronic marketplaces [1].

The contribution of this paper is a brief overview of the e-service vision and a component model for e-business applications based on the concept of service modules. We first describe the process-oriented approach we took to service specification. We then present the EJB-based (Enterprise Java Beans) prototype for an application platform based on service modules.

\section{E-Services Vision}

Until recently, the Internet was about the creation of ebusiness and e-commerce systems, and it was dominated by web sites, portals and store fronts. We have now entered the next Internet evolution: the proliferation of e-services. E-services are modular, nimble, electronic services that perform work, achieve tasks, or complete transactions [8]. Almost any asset can be turned into an e-service and offered efficiently via the Internet to drive new revenue streams. Chapter 1 of the Internet was about businesses getting wired to their employees, customers and partners; key business processes getting linked to the Internet, and a critical mass of consumers coming online. Businesses were learning how to use what looked like a promising new tool. 
Now, the Internet is ready for its next evolution. It will not be about businesses looking at the web as a technology. The Internet has been absorbed into the core business infrastructure, and businesses are ready to capitalise on this new asset. Chapter 2 of the Internet will be about the mass proliferation of e-services. An e-service is any asset that is made available via the Internet to drive new revenue streams. These services will be modular units that combine and recombine to solve problems, complete transactions, and make life easier. Some will be available on web sites, but others will be delivered via TV, phone, pager, car, email in-box, or virtually anything with a micro chip in it. Some will even operate behind the scenes, automatically working on behalf of consumers and providers.

In Chapter 2, successful companies have to be able to turn their assets into services delivered via the Internet. They will adopt an entrepreneurial approach to looking at their assets and determine how to leverage not only their core business offerings, but also their proprietary processes, data, relationships, knowledge and experience. In Chapter 2, we will see more companies turn these assets into services and offer them via the Internet.

\subsection{Use of Context for Service Components}

The first step to turn an existing asset or service into an e-service revolves around accessibility. The electronic virtualisation of the service has to provide communication channels that support automated conversational capabilities. Automation is fundamental at each step of the service delivery chain. Beyond the basic capability to exchange electronic messages using standard protocols on top of, e.g. an XML transport, the business logic behind the service provision and partner interaction has to be enforced. For example, the service offer has to be presented in a way that allows automated discovery to take place. The service description should enable advanced offer-request matching (beyond the basic pricing), as well as automated negotiation on contractual terms and parameters. The role of advanced directory services (e.g. UDDI), and in particular of electronic marketplaces is fundamental. An e-service is not a standalone entity but rather a first-class citizen of a highly dynamic ecosystem enabled by e-marketplaces.

The second step towards the realisation of the full potential for the e-service vision focuses on composition and interaction orchestration. An e-service has to expose all the interaction processes involved in the service delivery. Far from saying that a company should expose its core competences, the requirement is to handle the internal and external business networks dynamically created by every instance of service delivery. A service delivery may no longer be a one-to-one (buyer-to-seller) relationship. As an example, let us assume that the company iBuild has selected the company iMove for a shipment contract. The final product of iBuild may be packaged by a company iPack, and iBuild may want iMove to interact with iPack for arranging the logistics behind collecting the goods. Similarly, iMove's operational structure may be such that it focuses on hubto-hub transport using lorries, and it relies on partners for the hub-to-customer transport. In the case of the service sold to iBuild, iMove may select (directly or using an emarketplace) a company iVan to do the last leg of transport. As a consequence, iVan has to synchronise with iBuild and iPack. The responsibility to the end customer will be with iBuild in the same way as the overall responsibility for the end-to-end transport will be with iMove.

Thus e-service composition pulls multiple parties together. Some of them know some of the others, but in some cases the service providers might not be related. From an operational point of view, an e-service should be able to cooperate with a dynamically selected mix of other e-services. This implies the capability to automatically verify the behavioural compatibility of the various execution processes, as well as the capability to adapt them in order to make cooperation possible.

\subsection{A Model for Service Components}

Our service component model is based on the ideas of functional incompleteness, multi-party orchestration, and dynamic service composition $[4,6]$. A service can be partially incomplete in terms of its implementation. Metainformation present in the electronic virtualisation layer for the service specifies the kind of support services needed, as well as the type of integration required to become fully functional. For an e-service, the focus moves from the access logic to the integration logic. The challenge for both service providers and service consumers is to adopt an integration model based on business roles and behavioural descriptions.

(a)

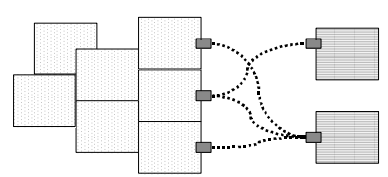

(c)

(b)
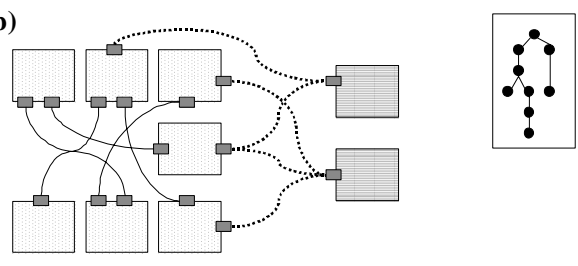

Figure 1: Process-based Interaction

In a business transaction, the service consumer has to be informed about the kind of interaction process supported 
by the service provider. The idea is to expose the service delivery process as early as possible, so that both service consumer and service provider can better evaluate their operational compatibility. The impact of e-services on the design for e-business systems is captured in Figure 1. Existing systems are developed around object-oriented models, and different functions are isolated into different parts of the system. The problem is (Figure 1a) that different functional modules are hardwired to each other in an ad-hoc way. The idea is instead to move to a scenario (Figure 1b) in which modules are kept separate. The interaction logic (Figure 1c) behind what then become service units is captured explicitly, and the distinction between internal and external service components is blurred.

The operational structure of a service can be designed with a new approach. First the need for specific support services is identified. Next the expected interaction processes with the potential service providers are identified. A specific service instance is available, only if adequate support services can be found. The concept of adequacy is heavily based on operational compatibility, in order to ensure a smooth execution of the overall service. The provider of an e-service component can focus on the implementation of the core aspects of the service. The e-service infrastructure will take care of the integration with the most suitable e-services to completely enable the new e-service. Integration logic coexists with business logic, still remaining two separate entities in terms of management and visibility.

\section{E-Service Beans}

As an implementation example of the component model proposed, we instrumented an EJB (Enterprise Java Beans [5]) platform with process-oriented componentisation capabilities [2]. The work revolved around the implementation of a new type of EJB container, within which a process description file can be used to model the observable behaviour of the bean. Clients and other beans will only be able to execute methods of a bean in this container if they are consistent with the process description. The outbound communication initiated by the bean is also monitored for compliance with the behavioural interface captured in the process. In-line with the naming conventions for EJB, we refer to the new container as ESB (E-Service Bean).

An E-Service bean represents a service unit, and the process description captures the service delivery process deriving from the external interaction of the bean. Different roles can be involved in the delivery process behind the service implementation. The ESB container manages at run-time the behaviour of the entities playing these roles. When a bean is created, the roles involved can be partitioned into groups and assigned transparently to either client programs or other beans. The only interaction allowed is the one deriving from the process description (both inbound and outbound). The aim of our prototype was to implement a basic container that demonstrates this kind of protection for the beans. The container that deploys service beans has the following features not found in normal EJB containers:

- The bean provider can specify the service behaviour in a process description file, that is then enforced by the container. The container generates exceptions whenever a method is called in an incorrect way (at the wrong point in the process or with invalid parameters).

- A process can be specified to have a number of roles that can be played. Clients can create a bean, specifying the roles they want to play, or contact an existing bean to have a roles assigned to them. Services cannot be started until all roles are assigned.

- The client can request role specific descriptions from a service bean to see what is required to do as the entity responsible for a specific roles.

- The system makes the state of each service instance persistent so that everything can be reconstructed in the event of a system crash.

The tasks performed by the system can be divided into two parts [5] that we discuss next. The first part is the creation of the home and remote object classes. The second part is the actual runtime handling of the beans.

Creating Home/Remote Objects: The archive containing interfaces, process description and deployment descriptor are made accessible to the container and deployed. The container then reads the manifest contained in the archive, finding the deployment descriptor. The deployment descriptor contains information on the persistent fields of the bean and whether the bean contains references to other service beans. If the bean does contain such references, then any method invocations made using the references will be checked against their behaviour specification. Once the information in the deployment descriptor has been read, the container can generate home and remote object classes. The home object is used by clients to create ESBs (partitioning the roles to be played into groups), request group specific remote objects, find remote objects, and destroy beans. The remote object is used by the client to make remote method calls on the bean.

Runtime Handling of the Beans: The second part of the EJB containers work involves making the home object available to clients and monitoring the use of the bean object via the home and remote objects. Once the home object is created it binds itself to the RMI Registry and makes 


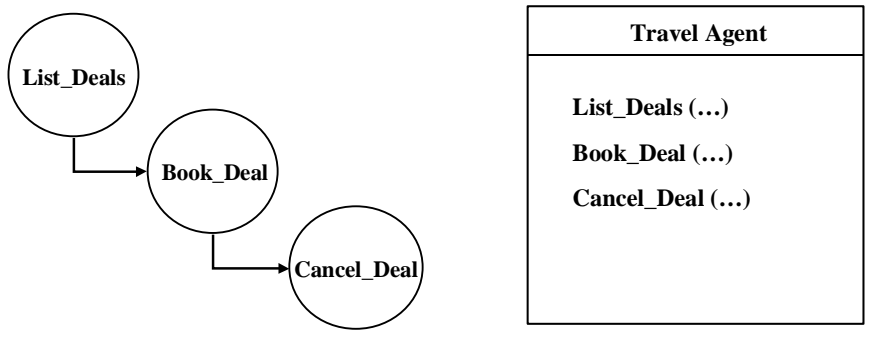

Figure 2: Customer View on Service Model

itself available via JNDI. Remote interaction between the client and the container takes place via Java RMI [3]. The client creating a service bean can dynamically partition the roles to be played by clients into groups. Each client plays a specific group of roles, and it receives a remote object used to call methods on the bean. The client that creates the service bean gets a remote object for the first group of roles to be played. The other clients are assigned groups using the assign method in the home object. The home object also creates a processutility object when a service bean is created and checks with the process description that the grouping of the roles it has been given by the home is correct. The remote object then uses the processutility object to check the validity of method calls. The remote objects can catch method invocations and return types to check that they are consistent with the process description. If they are not, a specific exception is thrown, otherwise the result is then returned to the client.

\section{ESB Container Application Scenario}

The activity of the ESB container is illustrated in a scenario consisting of three simple service beans, the Travel Agent bean, the Airline bean and the Hotel bean. The scenario shows the impact of external management on the interaction behaviour of the various components. In the scenario (Figure 3), a client first interacts with the travel agent to list holiday deals. The client books a holiday deal and then cancels it. Everything is coherent with the behavioural model specified by the travel agent. When the client books a holiday, the travel agent interacts with an airline and a hotel to book a flight and a hotel room for that holiday. The client can directly refer to the same airline to request information on "special deals", which are budget flights with a price of less than GBP 300. Though it is one of the methods exposed be the airline, the client is not allowed to do direct booking with the airline. When the client attempts the booking, the incompatibility between request and behavioural specification for the service is detected and the request is rejected.

The behavioural interface of the travel agent bean (Figure 2) specifies that this bean can be created and used by one entity only, which means there is only one free role available to be played. In the animation of our scenario, the client plays this role. The ESB container prevents other method invocations from reaching the beans, as they do not conform to the specified behaviour of the components. The Airline bean behavioural interface specifies two free roles, one of which will be played by the client and the other by the Travel Agent bean. The role played by the client will allow only the special_deals method to be called. The role played by the travel agent allows the invocation of the book_flight method. The Hotel bean has only one free role, which is played by the Travel Agent and can be used to book rooms. Figure 3 shows a basic client console and the monitor interface for the beans. The snapshot is taken immediately after the container has trapped a method invocation for the booking attempt from the customer to the airline. In this case the client has tried to invoke the book_flight method of the Airline bean, which is not available within the role the client is playing. The Airline bean is automatically shielded from the illegal request by the ESB container. Previous to the intercepted method invocations, the client called the list_deals.

\section{Related Work}

The CORBA 3.0 Component Model (CCM) [7] overcomes some of the deficiencies that we have identified for distributed objects. In particular, they avoid the hard-wiring of clients to server objects by introducing ports and receptacles along which components can publish and subscribe to events. The CCM, however, does not specify the ability to invoke operations and the value of parameters that need to be passed. Such concerns could be expressed in CORBA to some extent using the CORBA Security service [6], system-level access control policies can be used to express the privileges that are needed to be able to invoke certain operations and application-level access control may be used to determine which rights are needed to invoke operations with certain parameters. We however, strictly separate the definition of services from the way they may be combined. We can also specify certain precedence relationships and workflows, which cannot be specified by the security service. We note that our model of locating service beans using JNDI might be overly restrictive. In particular, it demands that a client or entity beans knows the name of the service bean from which it requires a service. We are currently investigating how our service model can be integrated with the ODP trading function or its implementation with the CORBA Trading service [6]). This will enable to specify a client a type of service and some qualities for service provision and then delegate to a trader to locate a suitable service bean that provides the required service. 


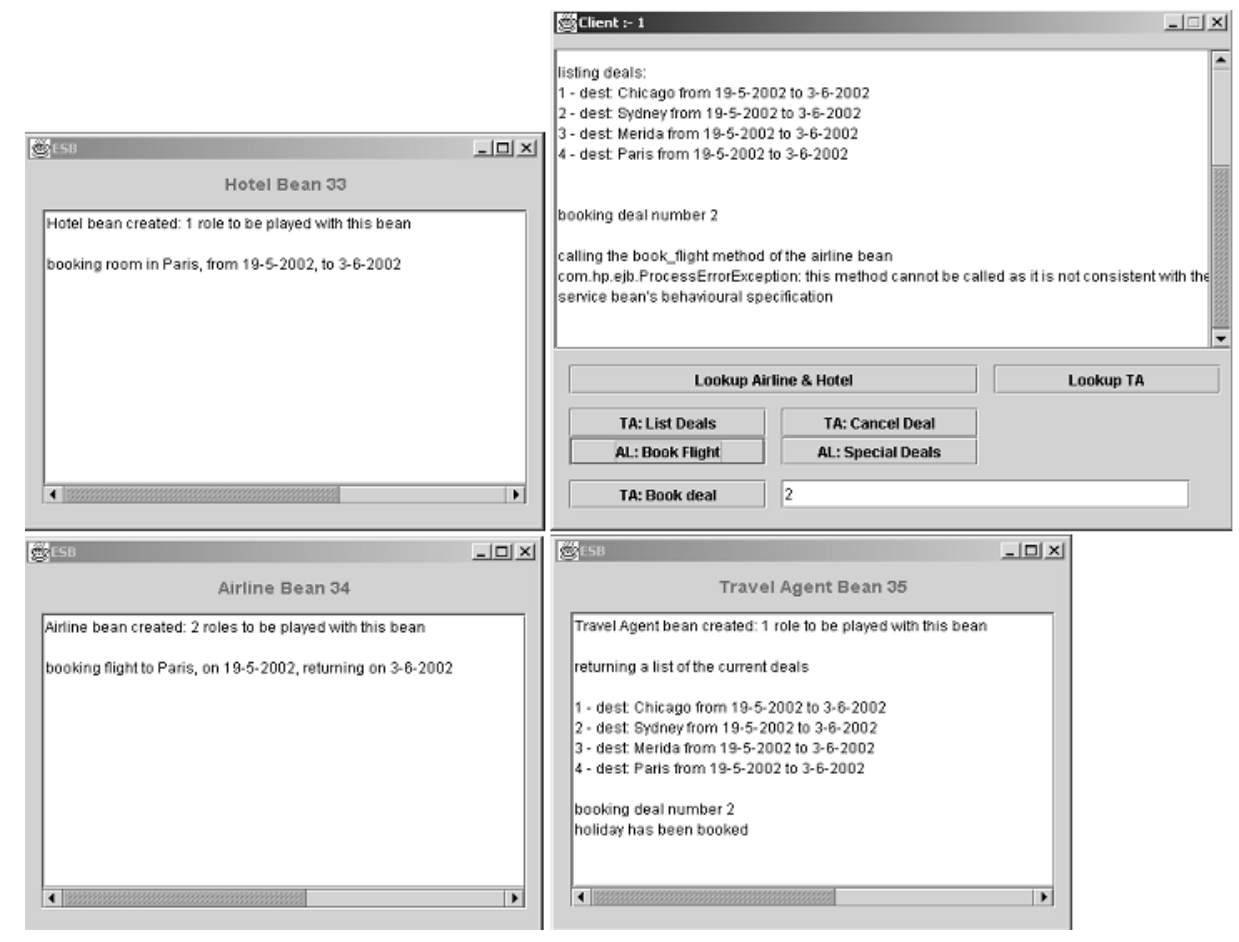

Figure 3: Component interaction mediated by ESB containers

\section{Conclusions}

E-business models often focus on the flexibility of the service offer. The capability to efficiently acquire the external resources required to satisfy specific demands is important, and electronic marketplaces play a key role in this process. Still, the quality and profitability of the service offer depends on the effective integration of external resources with internal business infrastructure. We propose that a serviceoriented modularisation of e-business systems could reduce the gap between internal and external components behind a service implementation. Based on the e-service vision, we propose a process-oriented model for the operational description of service components. Together with the foundational aspects of our proposal, in this paper we present a prototype infrastructure that instruments an EJB-platform with capabilities for the definition and implementation of e-service components.

\section{References}

[1] H. Blodget and E. McCabe. The B2B Market Maker Book. Merill Lynch \& Co, 2000.

[2] D. Holligsworth. The Workflow Reference Model. Technical Report TC00-1003, Workflow Management Coalition (WfMC), 1994.
[3] JavaSoft. Java Remote Method Invocation Specification, revision 1.50, jdk 1.2 edition, Oct. 1998.

[4] A. Marton, G. Piccinelli, and C. Turfin. Service Provision and Composition in Virtual Business Communities. In Proc. of the $18^{\text {th }}$ IEEE-IRDS Int. Workshop on Electronic Commerce, Lausanne, Switzerland, 1999.

[5] R. Monson-Haefel. Enterprise Javabeans. O'Reilly UK, 1999.

[6] Object Management Group. CORBAservices: Common Object Services Specification, Revised Edition. 492 Old Connecticut Path, Framingham, MA 01701, USA, December 1998.

[7] Object Management Group. CORBA Components - Volume I. 492 Old Connecticut Path, Framingham, MA 01701, USA, December 1999.

[8] H. Packard. E-Services. http://e-services.hp.com, 1999.

[9] D. G. Schwarz. Cooperating Heterogeneous Systems. Kluwer, 1995. 